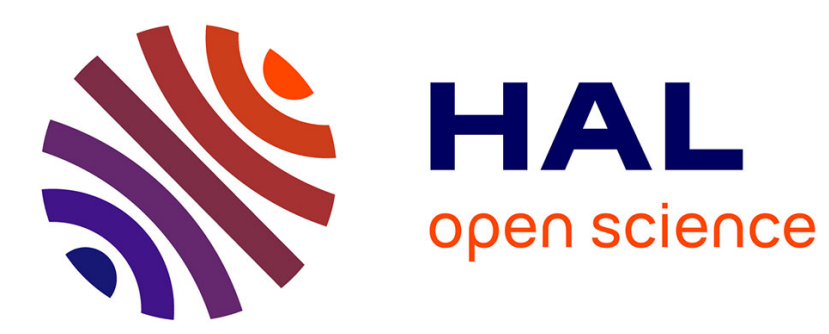

\title{
Divergence of the Stark collision operator at large impact parameters in plasma spectroscopy models
}

Joël Rosato, Hubert Capes, Roland Stamm

\section{To cite this version:}

Joël Rosato, Hubert Capes, Roland Stamm. Divergence of the Stark collision operator at large impact parameters in plasma spectroscopy models. Physical Review E : Statistical, Nonlinear, and Soft Matter Physics, 2013, Vol. 88, p.035101. hal-00945452

\section{HAL Id: hal-00945452 \\ https://hal.science/hal-00945452}

Submitted on 19 Feb 2014

HAL is a multi-disciplinary open access archive for the deposit and dissemination of scientific research documents, whether they are published or not. The documents may come from teaching and research institutions in France or abroad, or from public or private research centers.
L'archive ouverte pluridisciplinaire HAL, est destinée au dépôt et à la diffusion de documents scientifiques de niveau recherche, publiés ou non, émanant des établissements d'enseignement et de recherche français ou étrangers, des laboratoires publics ou privés. 


\title{
Divergence of the Stark collision operator at large impact parameters in plasma spectroscopy models
}

\author{
J. Rosato, H. Capes, and R. Stamm* \\ Aix-Marseille Université, CNRS, PIIM UMR 7345, F-13397 Marseille Cedex 20, France
}

\begin{abstract}
The divergence that occurs at large impact parameters in Stark collision operators is examined for low-density hydrogen plasmas. In a previous work [Rosato, Capes, and Stamm, Phys. Rev. E 86, 046407 (2012)], we showed that the correlations between a radiating atom and the charged particles surrounding it affect the mean evolution of the atom, resulting in a mitigation of the Stark broadening near the line center. In this work, we examine the physical mechanism underlying this mitigation with an approach inspired from the standard semi-classical impact model. Our approach accounts for the atom-perturber correlations in a simple fashion, through a cut-off at large impact parameters, and embraces the impact model in the weakly coupled plasma limit. Comparisons with numerical simulations are performed and indicate a good agreement.
\end{abstract}

PACS numbers: $32.70 . \mathrm{Jz}, 32.60 .+\mathrm{i}, 52.25 . \mathrm{Dg}, 52.70 . \mathrm{Kz}$

Recent theoretical works carried out in the framework of magnetic fusion research have led to successive improvements on ion collision operator models, devoted to provide fast numerical routines for hydrogen line shapes, for diagnostic applications but also for radiative transfer simulations (e.g. [1]). Although the ion Stark broadening is strongly dynamic for lines with a low upper principal quantum number $n$ (such as Ly- $\alpha, \mathrm{D}-\alpha$ ), the impact approximation for ions is only marginally valid and its use can lead to significant mistakes, with an overestimate of the line width at large densities or low temperatures (typically when $N_{e, i} \gtrsim 10^{14} \mathrm{~cm}^{-3}$ and/or $T_{e, i} \lesssim 1 \mathrm{eV}$, e.g. [2]). The inadequacy of the impact approximation rests in several assumptions that are not fulfilled. This is sometimes referred to as the "static effects". One such an effect concerns the reduction of the atom-perturber interaction to a duration of the order of $|\Delta \omega|^{-1}$ (with $\Delta \omega$ being the frequency detuning), which is effective as soon as $|\Delta \omega|$ exceeds the plasma frequency $\omega_{p}$ of the perturbing species. The latter corresponds to the characteristic time scale for an individual collision given the Debye shielding. Incomplete collisions have been investigated in the past for the electron broadening by using refined models for the collision operator, either based on kinetic theory (such as the "unified theory" $[3,4]$ ) or semiempirical procedures (e.g. using the Lewis cutoff [5]). Such models reproduce the result of the impact approximation at the line center (i.e., with the frequency detuning $\Delta \omega=0$ ) and yield an asymptotic behavior identical to that expected within the quasi-static approximation, which assumes a constant electric field. Another failure of the impact approximation concerns the line center in moderately coupled plasma conditions. The typical time for the atom's dipole decorrelation (estimated as the inverse of the collision operator's characteristic matrix elements) can be shorter than $\omega_{p}^{-1}$, yielding a re-

*Electronic address: joel.rosato@univ-amu.fr duction of the collision duration. Recently, we have examined this issue within a kinetic theory treatment inspired from the Bogoliubov-Born-Green-Kirkwood-Yvon hierarchy [6]. The reduction of the collision duration is a feature of emitter-perturber correlations and involves a new time scale which is in competition with the collision duration. In this Brief Report, we examine the physical mechanism underlying the emitter-perturber correlations with an approach inspired from the standard semiclassical impact model $[7,8]$.

We consider the mean evolution of an ensemble of emitters under the influence of the ion microfield. The ions are assumed statistically independent to each other, independent with respect to the electrons, and the electron perturbation is neglected. A "collision" refers to the perturbation of the atomic wavefunction due to one ion only. In the standard semi-classical impact model, it is customary to write down a finite difference equation for the atomic evolution operator and to average it along the following scheme

$$
\begin{aligned}
\{U(t+\Delta t, 0)\} & =\{U(t+\Delta t, t) U(t, 0)\} \\
& \simeq\left\{U_{j}(t+\Delta t, t)\right\}\{U(t, 0)\}
\end{aligned}
$$

Here, $U\left(t_{2}, t_{1}\right)$ stands for the evolution operator of the emitter from time $t_{1}$ to $t_{2}$, the brackets $\{\ldots\}$ denote average with respect to the ions, the time interval $\Delta t$ is chosen sufficiently large so as to include one collision only, and $U_{j}$ refers to the evolution of the emitter under the influence of this collision, which is labeled by $j$ (this includes the time of closest approach, the impact parameter, and the velocity). The average factorization stems from the independent ion assumption. In the standard impact model, the collisions are assumed complete during $\Delta t$, so that the evolution operator $U_{j}(t+\Delta t, t)$ is replaced by $U_{j}(+\infty,-\infty)$. This quantity is identical to the $S$-matrix used in the theory of collisions, hence, we will refer to it as $S_{j}$ in the following. The finite difference Eq. (1) for the mean evolution operator $\{U(t)\}$ becomes 
a differential equation at the limit $\Delta t \rightarrow 0$

$$
\frac{d\{U(t)\}}{d t}=\left(-i L_{0}-K\right)\{U(t)\}
$$

where $L_{0}$ is the atomic Liouvillian and $K=\sum_{j} \nu_{j}\left(1-S_{j}\right)$ is the collision operator, $\nu_{j}$ being the frequency of collision of type $j$. If the Stark effect is linear, the sum over collisions diverges logarithmically at large impact parameters. This result is a feature of the Coulomb interaction and is especially relevant for hydrogen lines. The usual way to prevent this divergence consists in putting an upper cut-off at the Debye length, accounting phenomenologically for the screening of the Coulomb field due to the other perturbers (which is not retained in the independent perturber assumption).

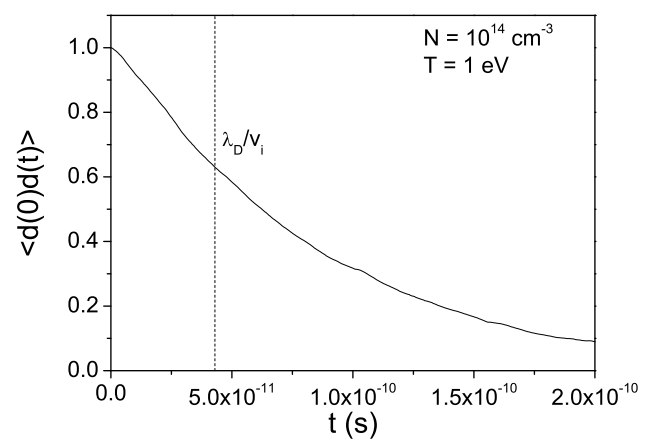

FIG. 1: Atomic dipole autocorrelation function of hydrogen Ly- $\alpha$ obtained from a simulation, at conditions relevant to tokamak edge plasmas. The collisions' effective duration is of the same order as the time of interest, suggesting an inadequacy of the complete collision assumption at the line center.

There is another regularization that prevents the divergence, which stems from the fact that each collision cannot be strictly completed, even at the line center. The collisions yield a decorrelation of the atomic dipole over a finite time scale $t_{i}$ (estimated as the inverse line width and usually referred to as the "time of interest"), which provides a maximum time for the effective action of each collision. Although this time can be much larger than the characteristic duration of each collision (of the order of $\omega_{p}^{-1}$ ), the dipole decorrelation should be retained in a realistic calculation. An illustration is given in Fig. 1. The microscopic electric field has been simulated at plasma conditions relevant to tokamak edge, during the time of interest for Ly- $\alpha$, assuming the ions moving along straight lines and generating a Debye electric field (due to the perturber-perturber correlations). For simplicity no magnetic field is considered here. The figure shows the dipole autocorrelation function obtained from numerical integration of the time-dependent Schrödinger equation, averaging with 1000 realizations of the electric field. Also shown in the figure is the effective duration of a collision, only slightly shorter than the time of interest.
In order to retain this regularization we extend the semi-classical impact model, by relaxing the complete collision assumption. We replace the $S$-matrix by the evolution operator $U_{j}\left(t_{j},-t_{j}\right)$ that accounts for one collision during a finite time. This time is described as a random variable distributed according to an exponential distribution of parameter $\gamma$, which is assumed of the order of the line width and is evaluated as some relevant matrix element of the collision operator (e.g., $\gamma \simeq\langle 200|K| 200\rangle$ for $\operatorname{Ly}-\alpha)$. With this model, the collision operator reads

$$
\begin{aligned}
K= & N \int_{0}^{+\infty} d v f(v) v \int_{0}^{+\infty} d b 2 \pi b \\
& \times \int_{0}^{\lambda_{D}} d t \gamma e^{-\gamma t}\{1-U(t,-t)\}_{\text {angle }} .
\end{aligned}
$$

Here, the $j$-index has been removed to clarify the notation, and the sum has been written explicitly in terms of integrals over the velocity (module) $v$ and the impact parameter (module) b. The brackets denote an angular average, and $N, f$ stand for the ions' density and their velocity distribution function, respectively. To illustrate the regularization we evaluate explicitly the cross section operator $\sigma=\int_{0}^{+\infty} d b 2 \pi b \int_{0}^{\lambda_{D}} d t \gamma e^{-\gamma t}\{1-U(t,-t)\}_{\text {angle }}$ and focus on the weak collision contribution, i.e., that provided by the second order expansion of the evolution operator. The calculation is similar to that performed in the standard impact model and it leads to the following analytical expression

$$
\sigma=\left.\frac{4 \pi \hbar^{2}}{3 m_{e}^{2} v^{2}} \vec{r}^{2} \Lambda(x)\right|_{\gamma \lambda_{D} / v} ^{\gamma b_{s t} / v}
$$

where $\vec{r}$ is the position operator of the atomic electron (atomic units) restricted to the Liouville subspace of the line under consideration, $b_{\text {st }}$ is the strong collision radius, of the order of the Weisskopf radius $b_{W}=\hbar n^{2} / m_{e} v$, and $\Lambda$ is defined as an integral

$$
\Lambda(x)=\int_{x}^{+\infty} d y\left\{\frac{1}{y}+\operatorname{Im}\left[e^{i y} E_{1}(i y)\right]\right\} .
$$

This function is plotted in Fig. 2. It behaves as $-\ln x-$ $\gamma_{E}$ (with $\gamma_{E}$ being the Euler constant) at small $x$. The presence of the first exponential integral $E_{1}$ regularizes the integral for large $x$. The integral can be evaluated explicitly in terms of the sine and cosine integrals [9]:

$$
\Lambda(x)=-\operatorname{Ci}(x) \cos x-\operatorname{si}(x) \sin x .
$$

We have compared the model to the result of a simulation excluding strong collisions, i.e., the perturbers with an impact parameter smaller than $b_{\text {st }}$ have not been retained. For simplicity we have assumed all perturbers with the same velocity module, evaluated at the relative thermal velocity $v_{i}=\sqrt{4 T / m_{i}}$ (the factor 4 accounts for the reduced mass of the emitter-perturber system). Figure 3 shows the dipole autocorrelation function for 


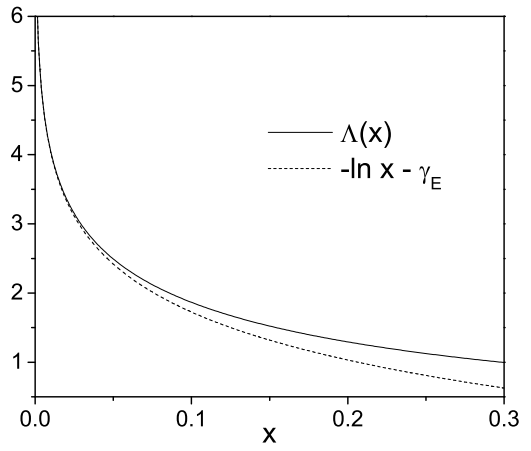

FIG. 2: Plot of the $\Lambda$ function. It has a logarithmic behavior at small $x$.

$N=10^{15} \mathrm{~cm}^{-3}$ and $T=1 \mathrm{eV}$, obtained from the simulation and from the model. In the latter case, it has been evaluated as $\left\langle 210\left|\exp \left(-N \sigma v_{i} t\right)\right| 210\right\rangle$. The $\gamma$ parameter has been estimated graphically from the simulation result, assuming an exponential decrease. Setting the equality $\left\langle d(0) d\left(\gamma^{-1}\right)\right\rangle=\exp (-1)$ yields $\gamma \simeq 4.2 \times 10^{10}$ $\mathrm{s}^{-1}$. The model provides a good estimate of the dipole decorrelation, with only a small deviation (by about $20 \%$ regarding the decay rate $N\langle 210|\sigma| 210\rangle v_{i}$ ) from the simulation, which falls in the typical range of uncertainties inherent to the description of the Debye screening and the estimate of strong collision radius. In contrast, the impact approximation leads to a strong deviation at large times. The faster decrease of the autocorrelation function stems from an overestimate of the Stark perturbation due to the complete collision assumption. This result is in agreement with that obtained from the kinetic theory treatment presented in [6].

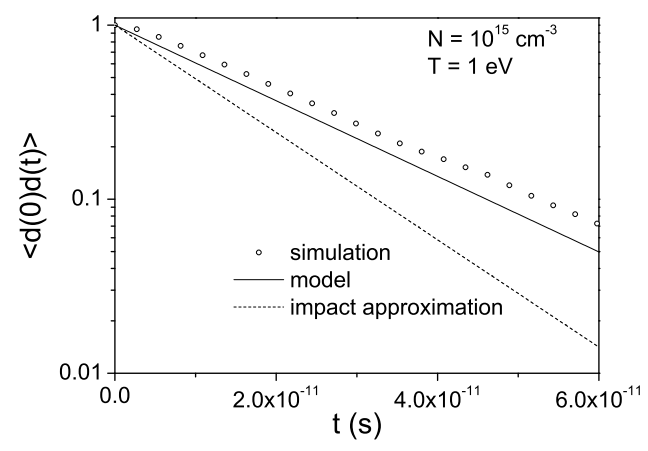

FIG. 3: Atomic dipole autocorrelation function of hydrogen Ly- $\alpha$ corresponding to perturbation by weak collisions. The complete collision assumption done in the impact approximation yields an overestimate of the Stark perturbation, which results in an overestimate of the decorrelation. The model proposed in this work is in a better agreement.
In summary, we have shown that the semi-classical impact approximation can be extended to account for incomplete collisions in a rather simple fashion, using an appropriate cut-off at large impact parameters. The resulting collision operator has a structure similar to that obtained within the usual impact model, making it suitable for implementation in line shape codes (e.g. [10-12]) and methods (e.g. [13-15]). The $\gamma$ parameter involved in the cut-off can be estimated from numerical simulations or analytically. In the latter case a characteristic matrix element of the collision operator was previously suggested as a good candidate [6]. The model is of particular interest for lines affected by linear Stark effect, given their sensitivity to the weak collisions. It can be applied to the ion broadening of hydrogen lines, in the case where ion dynamics effects are strong, but also to the electron broadening. This is of particular interest for lines with a high principal quantum number, in tokamaks but also in astrophysics. The recent Workshop on Spectral Line Shapes in Plasmas [16] provides a motivation to proceed with further investigations from the theoretical point of view.
This work was carried out within the framework of the European Fusion Development Agreement and the French Research Federation for Fusion Studies. It is supported by the European Communities under the contract of Association between Euratom and CEA. The views and opinions expressed herein do not necessarily reflect those of the European Commission. 
[1] D. Reiter, V. Kotov, P. Börner, K. Sawada, R. K. Janev, and B. Küppers, Journal of Nuclear Materials $\mathbf{3 6 3 3 6 5}$ 649 (2007).

[2] J. Rosato, Y. Marandet, H. Capes, S. Ferri, C. Mossé, L. Godbert-Mouret, M. Koubiti, and R. Stamm, Phys. Rev. E 79, 046408 (2009).

[3] D. Voslamber, Z. Naturforsch. 24a, 1458 (1969).

[4] E. W. Smith, J. Cooper, and C. R. Vidal, Phys. Rev. 185, 140 (1969).

[5] M. Lewis, Phys. Rev. 121, 501 (1961).

[6] J. Rosato, H. Capes, and R. Stamm, Phys. Rev. E 86, 046407 (2012).

[7] H. R. Griem, A. C. Kolb., and K. Y. Shen, Phys. Rev. 116, 4 (1959).

[8] H. R. Griem, M. Baranger, A. C. Kolb., and G. Oertel, Phys. Rev. 125, 177 (1962).

[9] M. Abramowitz and I. A. Stegun, Handbook of Mathe- matical Functions with Formulas, Graphs, and Mathematical Tables (Department of Commerce, Washington DC, 1964).

[10] S. Ferri, A. Calisti, C. Mossé, L. Mouret, B. Talin, M. A. Gigosos, M. A. Gonzáalez, and V. Lisitsa, Phys. Rev. E 84, 026407 (2011)

[11] R. C. Mancini, D. P. Kilcrease, L. A. Woltz, and C. F. H. Jr., Comput. Phys. Comm. 63, 314 (1991).

[12] M. L. Adams, R. W. Lee, H. A. Scott, H. K. Chung, and L. Klein, Phys. Rev. E 66, 066413 (2002).

[13] S. Alexiou, Phys. Rev. Lett. 76, 1836 (1996).

[14] S. Alexiou, High Energy Density Phys. 9, 375 (2013).

[15] E. Stambulchik and Y. Maron, Phys. Rev. E 87, 053108 (2013).

[16] E. Stambulchik, High Energy Density Phys. 9, 528 (2013). 